\title{
Semaphorin3D Regulates Axon-Axon Interactions by Modulating Levels of L1 Cell Adhesion Molecule
}

\author{
Marc A. Wolman, ${ }^{1,2}$ Ann M. Regnery, ${ }^{1}$ Thomas Becker, ${ }^{3}$ Catherina G. Becker, ${ }^{3}$ and Mary C. Halloran ${ }^{1,2}$ \\ ${ }^{1}$ Departments of Zoology and Anatomy and ${ }^{2}$ Neuroscience Training Program, University of Wisconsin, Madison, Wisconsin 53706, and ${ }^{3}$ Centre for \\ Neuroscience Research, Royal (Dick) School of Veterinary Studies, Summerhall, Edinburgh EH9 1QH, United Kingdom
}

\begin{abstract}
The decision of a growing axon to selectively fasciculate with and defasciculate from other axons is critical for axon pathfinding and target innervation. Fasciculation can be regulated by cell adhesion molecules that modulate interaxonal adhesion and repulsive molecules, expressed by surrounding tissues that channel axons together. Here we describe crosstalk between molecules that mediate these mechanisms. We show that Semaphorin3D (Sema3D), a classic repulsive molecule, promotes fasciculation by regulating L1 CAM levels and axon-axon interactions rather than by creating a repulsive surround. Knockdown experiments show that Sema3D and L1 genetically interact to promote fasciculation. Sema3D overexpression increases and Sema3D knockdown decreases levels of axonal L1 protein. Moreover, excess L1 rescues defasciculation caused by the loss of Sema3D. In vivo time-lapse imaging reveals that Sema3D or L1 knockdown cause identical defects in growth cone behaviors during axon-axon interactions, consistent with a loss of adhesion. These results reveal a novel mechanism by which a semaphorin promotes fasciculation and modulates axon-axon interactions by regulating an adhesion molecule.
\end{abstract}

Key words: semaphorin; L1; axon guidance; fasciculation; adhesion; zebrafish

\section{Introduction}

The formation of functional neural networks depends on the proper guidance of axons to their targets. A critical aspect of axon pathfinding is the decisions axons make to fasciculate with and defasciculate from particular axon tracts en route to their targets. In some cases, axons require fasciculation with a prelaid tract to reach their target area (Bastiani et al., 1984; Raper et al., 1984; Kuwada, 1986; Klose and Bentley, 1989), whereas in others, neighboring axons must coordinate with each other and segregate into specific fascicles to reach their proper targets (Tosney and Landmesser, 1985a,b; Lin et al., 1994). Moreover, suppressing axon-axon interactions to initiate defasciculation at specific choice points can be essential for target innervation (Tang et al., 1994; Yu et al., 1998, 2000). Thus, regulating axon-axon interactions is critical for the establishment of proper neural connections.

Axon-axon interactions are regulated by signaling cues, expressed on axons and in the surrounding tissue, that direct fasciculation and defasciculation by balancing adhesion and repulsion. Cell adhesion molecules (CAMs) can govern selective fasciculation decisions by regulating interaxonal and axon-

Received April 17, 2007; revised June 21, 2007; accepted July 14, 2007.

This work was supported by National Institute of Neurological Disorders and Stroke (NINDS) Grant NS042228 (M.C.H.), National Institute of General Medical Sciences National Research Service Award (NRSA) T32 GM07507 and NINDS NRSA 1F31NS054430 (M.A.W.). The National Science Foundation supported acquisition of the confocal microscope (Grant NSF9724515 to Jim Pawley, Department of Zoology, University of Wisconsin). We thank Dr. Wataru Shoji for providing the Neuropilin1A construct and Dr. Anand Chandrasekhar for providing the TAG-1 construct.

Correspondence should be addressed to Mary C. Halloran, Department of Zoology, 1117 West Johnson Street, Madison, WI 53706. E-mail: mchalloran@wisc.edu.

D0I:10.1523/JNEUROSCI.1741-07.2007

Copyright $\odot 2007$ Society for Neuroscience $\quad$ 0270-6474/07/279653-11\$15.00/0 substrate adhesion. For example, differential levels of Fasciclin II on motor neurons influence fasciculation and defasciculation events required for proper fascicle sorting (Lin and Goodman, 1994; Yu et al., 2000). Similarly, spatial and temporal modulation of neural cell adhesion molecule activity by polysialic acid regulates sorting of motor axons during limb innervation (Tang et al., 1992, 1994). Reducing interaxonal affinity or increasing the affinity of an axon for other substrates can induce defasciculation for target innervation (Kaneko and Nighorn, 2003; Chen et al., 2004). Expression of repulsive guidance molecules by the surrounding tissue also influences fasciculation. For example, semaphorins and slits are thought to drive fasciculation by creating a repulsive surround that channels axons into bundles and prevents their straying into nontarget tissues (Kitsukawa et al., 1997; Taniguchi et al., 1997; Isbister et al., 1999; Giger et al., 2000; Ringstedt et al., 2000; Cloutier et al., 2002, 2004; Hutson and Chien, 2002; Oster et al., 2003; Huber et al., 2005). However, we previously showed that knockdown of a repulsive semaphorin caused aberrant defasciculation of axons that are not normally bounded by its expression (Wolman et al., 2004), suggesting that semaphorins might also promote fasciculation via an unidentified mechanism.

We investigated mechanisms of axon fasciculation using a simple axon tract in the zebrafish brain, the medial longitudinal fascicle (MLF), as a model system. We provide evidence that Semaphorin3D (Sema3D) regulates axonal L1 protein levels to promote axon-axon interactions critical for fasciculation, rather than driving fasciculation by creating a repulsive surround. Sema3D and L1 are both required for MLF fasciculation and genetically interact with one another. Interestingly, Sema3D overexpression increases and Sema3D knockdown reduces ax- 
onal L1 protein levels. Excess L1 rescues defasciculation caused by loss of Sema3D, supporting the idea that Sema3D regulates L1 to promote MLF fasciculation. In vivo time-lapse imaging reveals defects in MLF axon-axon interactions after Sema3D or L1 knockdown that are consistent with a loss of adhesion. Similarly, Sema3D and L1 appear to regulate interactions between MLF and specific hindbrain axons that travel along the MLF. Finally, we show that Sema3D overexpression can cause hyperfasciculation of and increase L1 protein levels on axons that are normally attracted to Sema3D. Together, these results support a novel mechanism by which a secreted semaphorin promotes fasciculation by regulating an adhesion molecule to modulate axon-axon interactions.

\section{Materials and Methods}

Animals. Zebrafish (Danio rerio) were maintained in a laboratory breeding colony on a 14/10 h light/dark cycle. Embryos were maintained at $28.5^{\circ} \mathrm{C}$ and staged as described previously (Kimmel et al., 1995). The $h u c$ :green fluorescent protein $(g f p)$ stable transgenic line was created by Park et al. (2000).

Immunohistochemistry. For whole-mount immunohistochemistry, embryos were fixed in $4 \%$ paraformaldehyde overnight, blocked in 5\% sheep serum and $2 \mathrm{mg} / \mathrm{ml} \mathrm{BSA}$ in PBS with Triton X-100, and incubated overnight $\left(4^{\circ} \mathrm{C}\right)$ in the monoclonal antibody ZN-12 (1:250; Zebrafish International Resource Center, Eugene, OR) or anti-acetylated $\alpha$-tubulin (1:1500; Sigma, St. Louis, MO). Antibody labeling was performed with the Vectastain Mouse IgG ABC immunoperoxidase labeling kit (Vector Laboratories, Burlingame, CA). For fluorescent double labeling, embryos were simultaneously incubated with an anti-L1.1 (1:1000) (Becker et al., 2004) and either ZN-12 or anti-acetylated $\alpha$-tubulin. Alexa-conjugated secondary antibodies $(4 \mu \mathrm{g} / \mathrm{ml}$; Invitrogen, Carlsbad, CA) were used to bind primary antibodies.

In situ hybridization. Digoxygenin-UTP-labeled riboprobes for L1.1 and $L 1.2$ were synthesized by in vitro transcription and hydrolyzed to an average length of $\sim 300$ bases by limited alkaline hydrolysis (Cox et al., 1984). Whole-mount in situ hybridization was performed as described previously (Halloran et al., 1999).

Sema3D overexpression. Sema3D was overexpressed ubiquitously using a stable transgenic line in which the heat-inducible zebrafish heat shock protein $70(h s p 70)$ promoter drives expression of sema3D $[\mathrm{Tg}(\mathrm{hsp}$ : sema3 $D^{m y c}$ )] (Liu et al., 2004). Mosaic Sema3D overexpression was performed by injection of an $h s p: s e m a 3 D-g f p$ DNA construct at the $1-4$ cell stage. Transgene expression was induced by incubation at $39^{\circ} \mathrm{C}$ for $1 \mathrm{~h}$ ("heat shock") at relevant ages for analysis of MLF or anterior commissure (AC) axons.

mRNA overexpression. $5^{\prime}$ capped mRNA was transcribed from pcDNA3. $1^{+}$-neuropilin 1A (Nrp1A) (a gift from Wataru Shoji, Tohoku University, Sendai, Japan), $p C S 2^{+}$-transient axonal glycoprotein 1 (TAG-1) (a gift from Anand Chandrasekhar, University of Missouri, Columbia, MO), $p C S 2^{+}-G F P$, and $p c D N A 3.1^{-}-L 1.1$ using the mMessage mMachine kit (Ambion, Austin, TX). Fifty to $100 \mathrm{pg}$ of each RNA were injected into 1 cell stage embryos. Overexpression was verified for Nrp1A by in situ hybridization, GFP by fluorescence, and L1.1 and TAG-1 by in situ hybridization and immunohistochemistry at $24 \mathrm{~h}$ postfertilization (hpf).

Morpholino antisense. Morpholino oligonucleotides (MOs) against sema3D, nrp1A, L1.1, L1.2, plexinA3, and plexinA4 were synthesized by Gene Tools (Corvallis, OR). Sequences for morpholinos targeting Sema3D (Sema3DMO or Sema3D splice MO), Nrp1A (Nrp1AMO), PlexinA3 (PlexinA3MO1), PlexinA4 (PlexinA4MO), and control morpholinos (CONMO, STDCON) have been reported previously and tested for efficacy (Liu et al., 2004; Miyashita et al., 2004; Wolman et al., 2004; Liu and Halloran, 2005; Feldner et al., 2007). The sequences of morpholinos targeting L1.1 (L1.1MOA and L1.1MOB) and the L1.2 (L1.2MOA and L1.2MOB) morpholinos are as follows (with the sequence complementary to the start codon underlined or residue numbers in parentheses for sequences not overlapping with start codon):
L1.1MOA, 5' -CAGGCTGACTCTGCACTGGAGGCAT-3'; L1.1MOB, $(-13) \quad 5^{\prime}$-ATGAAAACAGCCCCGACTCCAGACA-3' ${ }^{\prime} \quad(-37)$; L1.2MOA， 5'-GCTGTTTTTGTGACGTGGCAGGCAT-3'; L1.2MOB, (-21) 5'-AACACAGCGGTGCAGGAAAG CCGTG-3' (-45). As a control for the L1MOs, we used the reverse sequence of L1.1MOA.

Morpholino oligos were injected into newly fertilized embryos at the 1-4 cell stage as described previously (Nasevicius and Ekker, 2000). Optimal concentrations for each $\mathrm{MO}$ were determined by titrating injection doses from 10 to $0.1 \mathrm{ng}$. The dose that caused robust defasciculation without nonspecific toxicity was used to elicit full knockdown. For subthreshold experiments, we chose the highest dose that did not cause significant defasciculation. The following amounts of each morpholino were injected to elicit full knockdown: $1 \mathrm{ng}$ of Sema3DMO or Sema3D splice MO, 1-3 ng of Nrp1AMO, 5-10 ng of L1.1MOs, 1-5 ng of L1.2MOs, 5 ng of PlexinA3MO1, and 5 ng of PlexinA4MO. Equivalent doses of appropriate controls were injected for each experiment, always on the same day. Subthreshold doses were as follows: $0.1 \mathrm{ng}$ of Sema3DMO, $0.5 \mathrm{ng}$ of Nrp1AMO, $1 \mathrm{ng}$ of L1.1MOA, and $0.5 \mathrm{ng}$ of L1.2MOA. For each experiment, gene-specific and the appropriate control MOs were injected into embryos obtained from the same spawning. For coinjection experiments (subMO/subMO, subMO/control, $\mathrm{MO} /$ mRNA, mRNA/control), we mixed the MOs/mRNA and injected them simultaneously. Injections were conducted at least three times for each experiment.

Western blot. Embryos were injected at the 1 cell stage with L1CONMO, L1.1MOA, L1.1MOB, L1.2MOA, or L1.2MOB and raised to $72 \mathrm{hpf}$ before homogenization. Protein extraction and Western blotting were performed with standard techniques. Blots were processed with anti-L1.1 or -L1.2 antibody and HRP-conjugated anti-rabbit IgG antibodies (Jackson ImmunoResearch, West Grove, PA) and visualized with chemiluminescence.

Imaging. All bright-field images were captured on a Nikon (Tokyo, Japan) TE300 inverted microscope equipped with a $40 \times$ [numerical aperture (NA) 0.75] or $60 \times(\mathrm{NA} 0.85$ ) objective and a Spot RT camera (Diagnostic Instruments, Sterling Heights, MI) and processed with MetaMorph software (Universal Imaging, West Chester, PA). Fluorescent images of fixed tissue are confocal projections captured on a Zeiss (Oberkochen, Germany) Axiovert 100M microscope with a $25 \times$ objective (NA 0.8) and the Bio-Rad (Hercules, CA) 1024 Lasersharp confocal. Step size was $1 \mu \mathrm{m}$.

In vivo time-lapse imaging. Preparation of embryos for imaging was adapted from Langenberg et al. (2003). Briefly, the yolk cell was paralyzed by adenosine $5^{\prime}$ ( $\beta$, $\gamma$-imido) triphosphate (Calbiochem, San Diego, CA) injection at $15.5 \mathrm{hpf}$ and then removed. Then, each embryo was mounted between two coverslips in $67 \%$ L-15. Embryos ranged in age from 16 to $17 \mathrm{hpf}$ at the start of imaging and were imaged for 2-12 h. Images were captured on a Nikon E-600FN equipped with standard epifluorescence, a $60 \times$ dipping objective (NA 1.00), a filter wheel, and a CoolSnap HQ camera (PhotoMetrics, Tucson, AZ). Images were captured every $1 \mathrm{~min}$, and exposure times were typically $300-600 \mathrm{~ms}$. Images and movies were processed and analyzed with MetaMorph software.

Quantification of L1.1 protein. Unsaturated, confocal projections of fluorescently labeled embryos with ZN-12 (or anti-acetylated $\alpha$-tubulin) and anti-L1.1 antibodies were analyzed with MetaMorph software. All imaging acquisition parameters, including laser intensity and pinhole size, were held constant across experiments. For each embryo, an image stack of $25 \mu \mathrm{m}$, including the entire thickness of the MLF, was projected into a single image using the average pixel intensity algorithm. To quantify L1.1 protein expression by MLF axons, we outlined the area occupied by ZN-12-labeled MLF axons from the caudal edge of the bilateral nuclei of the MLF (nucMLF) through rhombomere 3 and measured the average fluorescence intensity on the axons within the outlined region (supplemental Fig. 2, available at www.jneurosci.org as supplemental material), The outlined region was transferred to an anti-L1.1 antibody labeled projection of the same embryo to calculate the average intensity of antiL1.1 fluorescence. A ratio of the average intensity of L1.1 to the average intensity of ZN-12 was calculated to correct for any nonspecific fluctuations in the antibody labeling process. Outlines and intensity measurements were performed four times by two different experimenters (one 
A

Wild-type:
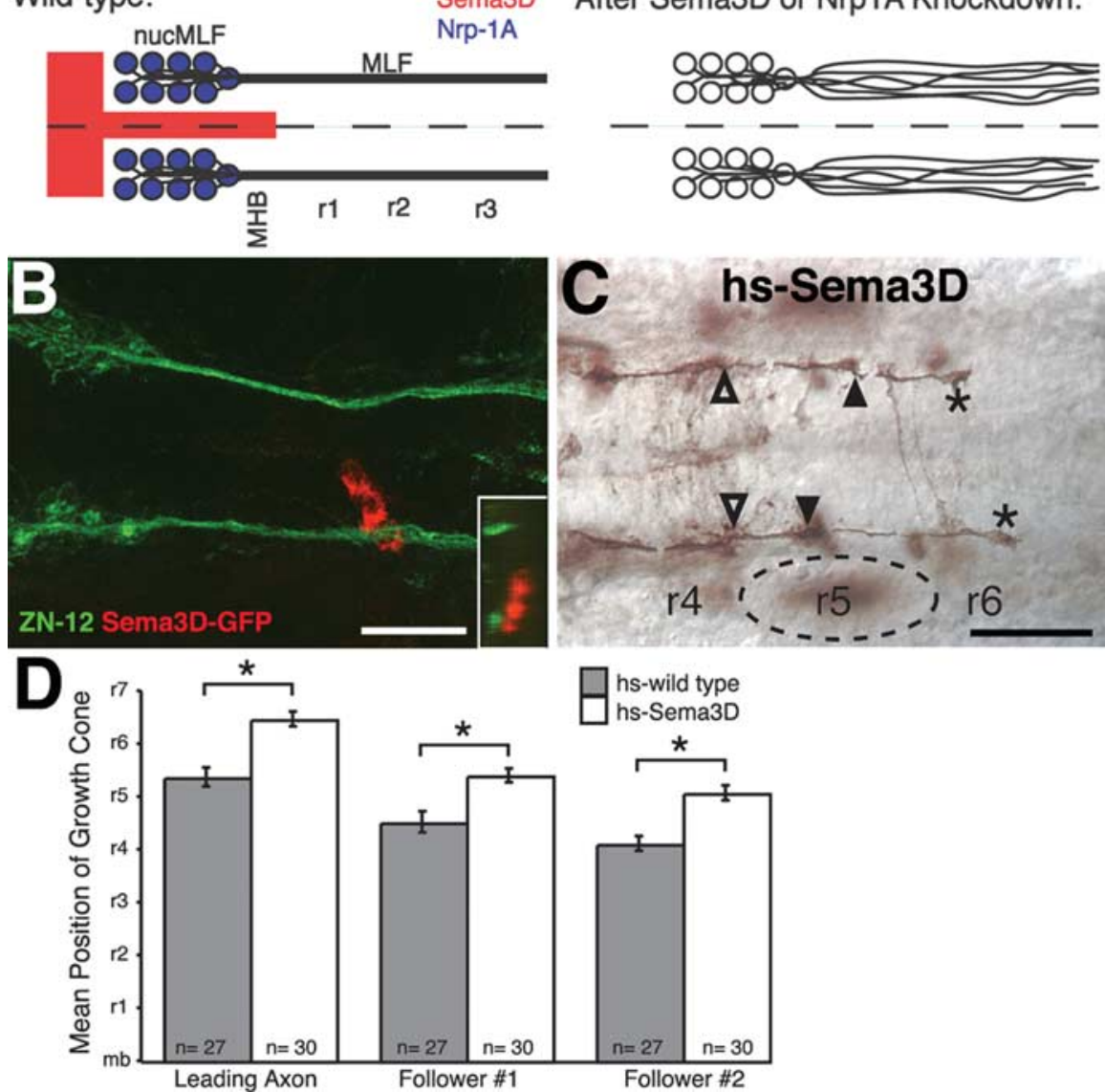

Figure 1. Sema3D does not repel MLF axons in the anterior hindbrain. $\boldsymbol{A}$, Schematic representation of sema3D expression (red) in relation to the nrp1A-expressing (blue) nucMLF and the defasciculation caused by knockdown of Sema3D or Nrp1A. MHB, Midbrain-hindrain boundary. $\boldsymbol{B}$, Confocal projection of $24 \mathrm{hpf}$ embryo injected with hsp:sema3d-gfp DNA construct and heat shocked to induce mosaic Sema3D-GFP expression. An embryo stained with anti-GFP (red) and ZN-12 (green) antibodies is shown. The inset shows $90^{\circ}$ rotation of Z-series. C, Heat-shocked $\mathrm{Tg}$ (hsp:sema3d ${ }^{\text {myc }}$ ) embryo labeled with ZN-12 at 19 hpf. Asterisks denote growth cone of leading MLF axon. The filled arrowhead and open arrowhead indicate growth cones of first and second follower MLF axons, respectively. The dashed oval represents perimeter of otocyst. $D$, Quantification of mean position of growth cones of MLF axons at $19 \mathrm{hpf}$. Each growth cone was given a numerical score based on rhombomere position (i.e., $\mathrm{r} 5=5$ ). ${ }^{*} p<0.001$, two-tailed $t$ test. Error bars represent SEM. Scale bars, $50 \mu \mathrm{m}$.

blind) and averaged. Mean L1.1/ZN-12 ratios were compared among groups using a two-tailed $t$ test. Anti-tubulin antibodies were also used as an additional marker to normalize L1.1 levels in MLF axons. The same procedures were applied for quantifying L1.1 levels in AC axons stained for L1.1 and anti-acetylated $\alpha$-tubulin. AC axons were outlined between each dorsal rostral cluster.

\section{Results}

Sema3D does not promote MLF fasciculation via repulsion The zebrafish MLF is a major longitudinal axon tract that extends between the midbrain and the spinal cord in the ventral neural tube. The MLF is initially formed by axons of the nucMLF, located in the ventral midbrain, that extend caudally through the hindbrain and into the spinal cord and serve as a scaffold on which multiple axon populations extend in the rostrocaudal axis. Previously, we showed that sema3D is expressed both rostral and medial to the nucMLF clusters before and during initial MLF axon extension (Halloran et al., 1999; Wolman et al., 2004) (Fig. 1A). Knockdown of Sema3D or its putative receptor component Nrp1A, which is expressed by the MLF neurons, causes MLF axons to defasciculate, indicating that Sema3D normally pro- motes MLF fasciculation (Wolman et al., 2004) (Fig. 1A). Given our previous characterizations of Sema3D as an axonal repellant (Liu et al., 2004; Wolman et al., 2004; Liu and Halloran, 2005) and other studies suggesting that repulsive class 3 semaphorins drive fasciculation by creating a repulsive surround (Kitsukawa et al., 1997; Taniguchi et al., 1997; Isbister et al., 1999; Giger et al., 2000; Cloutier et al., 2002, 2004; Oster et al., 2003; Huber et al., 2005), it is possible that Sema3D promotes MLF fasciculation via repulsion. Interestingly, however, defasciculated MLF axons in Sema3D knockdown embryos did not converge on the normal domain of sema3D midline expression, as might have been expected if Sema3D promoted MLF fasciculation via repulsion.

To address whether Sema3D is repulsive to MLF axons extending in the anterior hindbrain, we expressed Sema3D ectopically, either mosaically or ubiquitously, and assessed MLF axon outgrowth. Sema3D was expressed mosaically by injecting a DNA construct encoding GFP-tagged Sema3D under the control of an $h s p 70$ heat-inducible promoter ( $h s p$ : sema3d-gfp) into $1-4$ cell stage wild-type embryos, which were subsequently heat shocked to drive mosaic Sema3D expression during MLF axon outgrowth. We have shown previously that retinal ganglion cell axons and peripheral RohonBeard axons are repelled by cells expressing this construct (Liu et al., 2004; Liu and Halloran, 2005). Therefore, we hypothesized that, if Sema3D were repulsive to MLF axons in the anterior hindbrain, then an ectopic source of Sema3D located in the normal path of MLF axons would alter their trajectory. However, in embryos with Sema3D-GFP-expressing cells within 10 $\mu \mathrm{m}$ from the MLF, we never observed MLF axons diverting their trajectory to avoid the ectopic Sema3D $(n=15)$ (Fig. $1 B$ ). In fact, some fasciculated MLF axons even contacted the ectopic Sema3D-GFP-expressing cells (Fig. $1 B$, inset). We also hypothesized that, if Sema3D were repulsive to MLF axons in the hindbrain, then ubiquitous overexpression would stunt their outgrowth. To induce ubiquitous Sema3D overexpression, we used a stable transgenic line in which myc-tagged sema3D is driven by hsp70 [Tg(hsp:sema3D $\left.\left.{ }^{m y c}\right)\right]$ (Liu et al., 2004). We heat shocked age-matched $\operatorname{Tg}\left(h s p: s e m a 3 D^{m y c}\right)$ and wild-type embryos at 14 hpf and compared the position of their growth cones in the hindbrain at 19 hpf. In heat-shocked wild-type control embryos (hswild type), the growth cone of the leading axon was typically located in rhombomere 5 (r5), and the growth cones of the first and second follower axons had grown into r4 (Fig. 1D). However, in embryos overexpressing Sema3D (hs-Sema3D), the growth cone of the leading axon was in r6, and the growth cones of the first and second followers were in $\mathrm{r} 5$ (Fig. 1C,D), suggesting that Sema3D overexpression actually increased the MLF axon growth rate. Collectively, these data are inconsistent with 
Sema3D promoting MLF fasciculation via surround repulsion and suggest that Sema3D functions via an alternative mechanism.

\section{L1.1 genetically interacts with Sema3D and Nrp1A to promote MLF fasciculation}

Receptor complexes that mediate growth cone responses to class 3 semaphorins consist of a Neuropilin binding component, a Plexin signal-transducing component, and sometimes the CAM L1 (Castellani et al., 2000, 2002, 2004; Haspel and Grumet, 2003). Each component can also mediate cell-cell adhesion (Ohta et al., 1995; Fujisawa et al., 1997; Brummendorf et al., 1998; Shimizu et al., 2000). We investigated whether L1 mediates MLF fasciculation by Sema3D. Zebrafish have two L1 orthologs, L1.1 and L1.2, which are both expressed by the majority of neurons in the zebrafish brain, including the nucMLF (Tongiorgi et al., 1995) (Fig. 2A,B). Two nonoverlapping MOs were designed against each zebrafish L1 ortholog (L1.1MOA and L1.1MOB, L1.2MOA, and $\mathrm{L} 1.2 \mathrm{MOB}$ ), and the reverse sequence of L1.1MOA was injected as a control (L1CONMO). Both morpholinos specifically targeting each L1 ortholog, but not L1CONMO, inhibited protein translation of the appropriate L1 ortholog (supplemental

Fig. 1, available at www.jneurosci.org as supplemental material). In our initial quantifications of MLF defasciculation (see below), we pooled embryos injected with each gene-specific MO and refer to these pools as L1.1MO or L1.2MO. For subsequent experiments, we injected L1.1MOA or L1.2MOA and refer to them as L1.1MO or L1.2MO, respectively.

To determine whether either L1 ortholog was critical for MLF fasciculation, we injected embryos at the 1-4 cell stage with L1.1MOA, L1.1MOB, L1.2MOA, L1.2MOB, or L1CON and allowed them to develop to $24 \mathrm{hpf}$, when many MLF axons have grown through the anterior hindbrain. Injection of L1.1MOA or L1.1MOB each caused MLF defasciculation in $64 \%(n=42$ and 48 , respectively) of embryos, whereas injection of L1CONMO only caused defasciculation in 5\% $(n=110)$ of embryos (Fig. $2 C, D, G)$. L1.2MOA or L1.2MOB injection caused MLF defasciculation in $60 \%(n=30)$ and $76 \%(n=26)$ of embryos, respectively (Fig. 2G). These results suggest that both L1.1 and L1.2 are required for MLF fasciculation.

We showed previously that Sema3D and Nrp1A genetically interact to promote MLF fasciculation (Wolman et al., 2004) (Fig. $2 H$ ). To investigate whether L1 functions in the same pathway as Sema3D and Nrp1A to promote MLF fasciculation, we analyzed potential genetic interactions among these genes. If Sema3D acts via a particular L1 ortholog to regulate fasciculation, then partial knockdown of the L1 ortholog should enhance the very weak phenotype caused by partial knockdown of Sema3D. To test this idea, we performed coinjection experiments with subthreshold doses of morpholinos, in which we mixed subthreshold doses of two different morpholinos. We and others have used this method previously to show genetic interaction
(Wolman et al., 2004; Kuan et al., 2007). We defined the subthreshold dose as the highest dose that did not produce significant defasciculation. As controls, we injected subthreshold doses of the gene-specific morpholino plus enough standard control morpholino to make the final concentration equal to that injected for the coinjection groups. Simultaneous partial knockdown of Sema3D and L1.1 caused MLF defasciculation in 75\% $(n=67)$ of embryos compared with $6-19 \%$ of controls (Fig. $2 E, H)$. Furthermore, simultaneous partial knockdown of Nrp1A and L1.1 caused MLF defasciculation in $68 \%(n=77)$ of embryos compared with $14-19 \%$ of controls (Fig. $2 F, H$ ). These data suggest that Sema3D, L1.1, and Nrp1A function together to promote MLF fasciculation. In contrast, we did not observe a genetic interaction between L1.2 and Sema3D or Nrp1A (Fig. $2 H$ ). Given the high degree of sequence divergence between the L1 orthologs (Tongiorgi et al., 1995), it is possible that L1.2 has evolved to influence MLF fasciculation via a Sema3D-independent pathway. Thus, Sema3D, L1.1, and Nrp1A likely regulate MLF fasciculation through a common signaling pathway, but L1.2 does not appear to interact with Sema3D or Nrp1A to promote MLF fasciculation.

\section{Sema3D regulates $\mathrm{L} 1.1$ protein levels in MLF axons}

The genetic interaction suggests that L1.1 could be a receptor component necessary for mediating Sema3D repulsion. However, because our data suggest that Sema3D does not promote MLF fasciculation via repulsion, we explored the possibility that Sema3D regulates L1.1 protein levels on MLF axons to modulate their adhesive interactions. To test this idea, we measured L1.1 protein levels on MLF axons after Sema3D knockdown or over- 


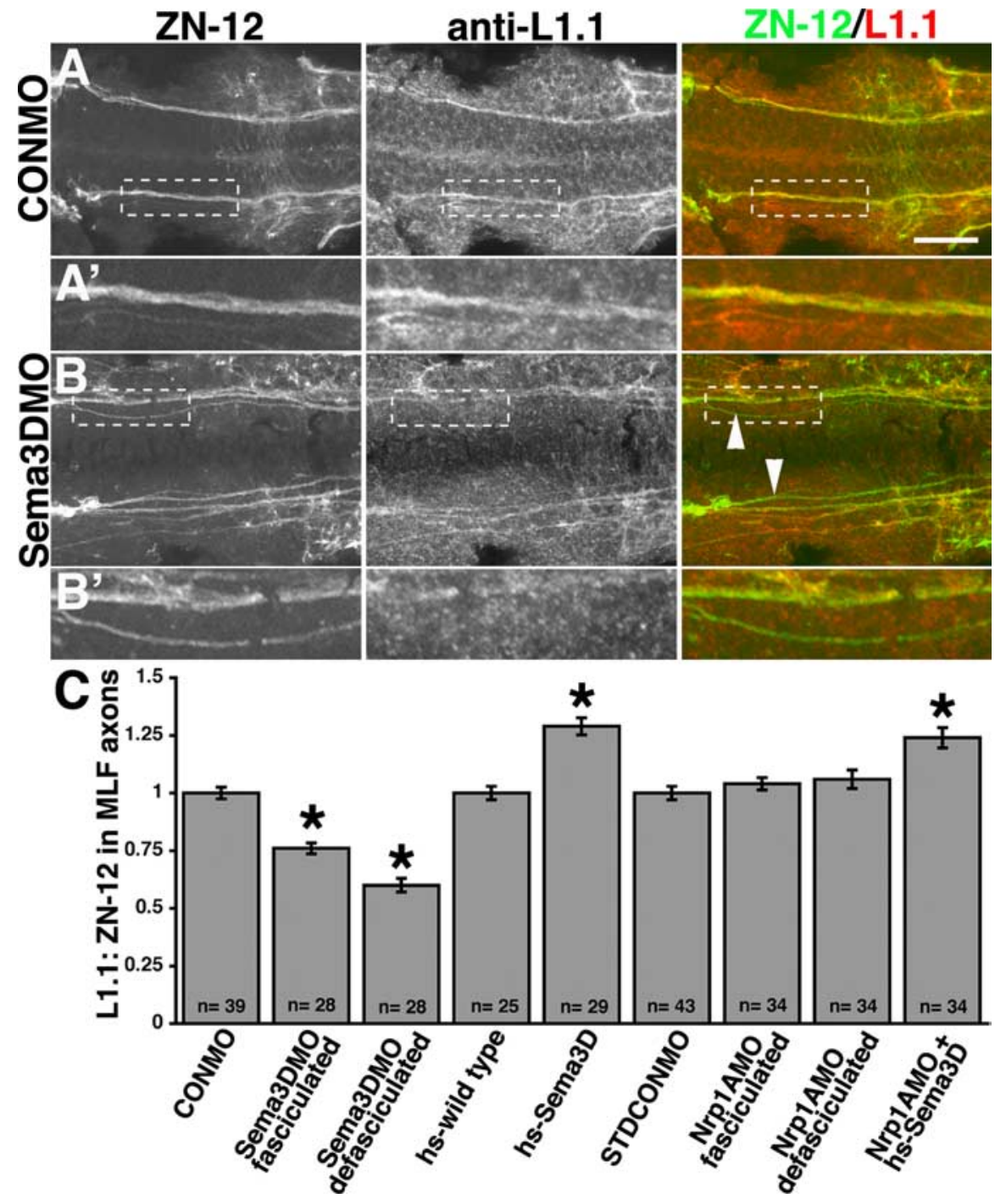

Figure 3. Sema3D regulates $L 1.1$ protein levels in MLF axons. $\boldsymbol{A}, \boldsymbol{B}$, Unsaturated confocal projections of $24 \mathrm{hpf}$ embryos, labeled with ZN-12 (green) and L1.1 (red) antibodies. Anterior is to the left. $\boldsymbol{A}, \boldsymbol{A}^{\prime}$, Four-mispaired base morpholino (vs Sema3DM0 sequence) injected embryo (CONMO). $\boldsymbol{B}, \boldsymbol{B}^{\prime}$, Sema3DM0-injected embryo; arrowheads indicate defasciculated MLF axons that express significantly less L1.1. $\boldsymbol{A}^{\prime}, \boldsymbol{B}^{\prime}$, Higher magnification of dashed-box area in $\boldsymbol{A}$ and $\boldsymbol{B}$, respectively. $\boldsymbol{C}$, Mean L1.1/ZN-12 ratio of fluorescence intensity in MLF axons. ${ }^{*} p<0.001$, two-tailed $t$ test. Error bars represent SEM. Scale bar, $50 \mu \mathrm{m}$.

ciculated (Fig. $3 B, B^{\prime}$ ). We measured the fluorescent intensity on individual defasciculated axons and found that they had a $40 \%$ decrease in L1.1/ZN-12 ratio compared with the fasciculated axons in control injected embryos (Fig. 3C). Axons that remained fasciculated in Sema3DMOinjected embryos had a $19 \%$ decrease in L1.1/ZN12 ratio compared with fasciculated axons in CONMO-injected embryos (Fig. $3 B, B^{\prime}, C$ ). Importantly, knockdown of Nrp1A did not significantly affect MLF L1.1 protein levels despite causing defasciculation (Fig. $3 C$ ), suggesting that defasciculation alone does not influence L1.1 levels or our intensity measurements. In contrast to Sema3D knockdown, ubiquitous Sema3D overexpression significantly increased L1.1 levels in MLF axons (Fig. $3 C)$. Heat-induced Sema3D overexpression in $\mathrm{Tg}\left(\right.$ hsp:sema3 $\left.\mathrm{D}^{m y c}\right)$ transgenic embryos (hs-Sema3D) increased the L1.1/ $\mathrm{ZN}-12$ ratio in MLF axons by $29 \%(n=$ 29) (Fig. 3C) compared with hs-wild type or unheated transgenic embryos (data not shown). For the above manipulations, similar results were obtained when an anti-acetylated $\alpha$-tubulin antibody was substituted for $\mathrm{ZN}-12$ as a normalizing marker (data not shown). Collectively, these data are consistent with our hypothesis that Sema3D regulates L1.1 protein levels on MLF axons to promote their fasciculation.

\section{Sema3D regulation of $\mathrm{L} 1.1$ is critical for} MLF fasciculation

Next we asked whether the main role of Sema3D in promoting MLF fasciculation is to maintain enough L1.1 protein to ensure an adequate degree of adhesion. To address this question, we tested whether excess L1.1 could compensate for the loss of Sema3D. We injected embryos with

expression. We colabeled embryos with anti-L1.1 and ZN-12 antibodies. L1.1 immunolabeling detected L1.1 protein in both neurons and more weakly in surrounding tissue (Fig. 3A,B), which we attributed to solubilized L1.1, because L1.1 mRNA has been detected exclusively in neurons and L1 protein can be cleaved extracellularly (Sadoul et al., 1988; Beer et al., 1999; Nayeem et al., 1999; Mechtersheimer et al., 2001). However, we measured L1.1 levels only on MLF axons (see Materials and Methods) (supplemental Fig. 2, available at www.jneurosci.org as supplemental material). We measured the average fluorescent intensity of each antibody in MLF axons from unsaturated confocal projections and calculated the intensity ratio of L1.1/ZN-12. ZN-12 intensity did not significantly fluctuate after genetic manipulation of Sema3D or Nrp1A (supplemental Fig. 2, available at www.jneurosci.org as supplemental material), nor did the apparent number of nucMLF cells or MLF axons. Sema3D knockdown caused reduced axonal L1.1 levels. Interestingly, we noticed that defasciculated MLF axons in Sema3DMO-injected embryos appeared to express less L1.1 protein than those that remained fas- both Sema3DMO and L1.1 mRNA and determined whether this rescued MLF defasciculation. Indeed, only 17\% $(n=52)$ of these embryos (Sema3DMO + L1.1 $\uparrow$ ) exhibited MLF defasciculation compared with $60 \%(n=72)$ of embryos injected with Sema3DMO and GFP mRNA (Fig. $4 A, B, D$ ). However, in the converse experiment, Sema3D overexpression did not rescue defasciculation caused by L1.1 knockdown (L1.1MO + hsSema3D) (Fig. 4C,D). We find that Sema3D overexpression, however, is sufficient to rescue defasciculation caused by the Sema3D splice-blocking MO (M. A. Wolman and M. C. Halloran, unpublished data). Because L1.1 is highly adhesive, its overexpression could potentially rescue defasciculation caused by Sema3D knockdown even if the two genes do not function together. Therefore, we attempted to rescue defasciculation caused by Sema3DMO knockdown by overexpressing TAG-1 (Sema3DMO + TAG-1 $\uparrow$ ), a glycosylphosphatidylinositollinked Ig superfamily CAM that is also highly adhesive (Karagogeos, 2003) via mRNA injection. We used this TAG-1 construct to rescue axon fasciculation defects caused by TAG-1 knockdown 
(Wolman and Halloran, unpublished data). However, TAG-1 overexpression did not rescue MLF defasciculation caused by Sema3D knockdown (Fig. 4D), suggesting that increasing the expression of any adhesion molecule is not sufficient to rescue MLF fasciculation in the absence of Sema3D. Likewise, overexpression of Nrp1A did not rescue defasciculation caused by L1.1 knockdown (L1.1MO + Nrp1A $\uparrow$ ) (Fig. 4D). Importantly, overexpression of Sema3D, L1.1, Nrp1A, or TAG-1 alone did not alter the normal fasciculation of MLF axons (data not shown). Together, these results suggest that proper L1.1 levels are critical for MLF fasciculation and that the role of Sema3D is to ensure an adequate level of L1.1.

Interestingly, either Sema3D or L1.1 overexpression was sufficient to rescue defasciculation caused by Nrp1A knockdown (Fig. 4D), suggesting that increased L1.1 levels may compensate for the loss of Nrp1A. Moreover, the ability of Sema3D overexpression to rescue Nrp1AMOinduced MLF defasciculation suggested the possibility that Sema3D might regulate L1.1 independently of Nrp1A. This would be surprising because Nrp1A is the only Nrp expressed in the zebrafish nucMLF, and our previous studies showed it is likely a receptor for Sema3D-induced repulsion (Wolman et al., 2004). To test this possibility, we injected $\mathrm{Tg}\left(\right.$ hsp:sema3 $\left.D^{m y c}\right)$ embryos with the Nrp1AMO, induced Sema3D overexpression, and colabeled MLF axons with the anti-L1.1 and ZN-12 antibodies to quantify L1.1 levels of these axons. Sema3D overexpression significantly increased L1.1 levels in MLF axons after Nrp1A knockdown $(n=19)$ (Fig. $3 C)$, suggesting that Sema3D regulation of L1.1 levels may be Nrp1A independent or at least does not require normal levels of Nrp1A.

In most known cases, class 3 semaphorins require an Nrp and a Plexin receptor component, but one important study showed that Sema3E can act directly through PlexinD1 and does not require a Nrp receptor component (Gu et al., 2005). We sought to identify a Plexin that is required for MLF fasciculation and that potentially mediates regulation by Sema3D of L1.1 levels. Zebrafish plexinD1 is expressed only in endothelial cells and is not in the nucMLF (Torres-Vazquez et al., 2004). However, two identified zebrafish class A Plexins, plexinA3 and plexinA4, are expressed in the nucMLF (Miyashita et al., 2004; Feldner et al., 2007). We injected morpholinos against PlexinA3, PlexinA4, or both at previously published full-strength doses (Miyashita et al., 2004; Feldner et al., 2007). However, neither knockdown of PlexinA3 or PlexinA4 individually nor combined knockdown of both caused significant MLF defasciculation (data not shown). These results suggest that PlexinA3 and PlexinA4 do not mediate MLF fasciculation, or, if one of them is a component of the Sema3D receptor, it may have a redundant function with other yet uncharacterized Plexins.

\section{Knockdown of Sema3D or L1.1 disrupts MLF axon-axon interactions critical for fasciculation}

To determine how Sema3D or L1.1 knockdown affects dynamic interactions between axons, we performed in vivo time-lapse imaging of MLF development. We hypothesized that, if Sema3D regulates MLF fasciculation by modulating L1.1 levels, then we might see effects on dynamic axon-axon contacts after knockdown of either gene. To visualize MLF growth cones, we used a stable transgenic line in which all neurons express GFP under control of the HuC promoter [Tg(huc:gfp)] (Park et al., 2000). First, we characterized growth cone behavior during normal MLF development in uninjected $T g(h u c: g f p)$ embryos and then in embryos injected with either Sema3DMO or L1.1MO. We began imaging at $\sim 16 \mathrm{hpf}$, when MLF axons initially extend from the nucMLF, and focused on the region spanning the caudal portion of the nucMLF through r1 (Fig. 5A).

In uninjected controls, the caudalmost nucMLF cell typically extended the leading MLF axon. Then, subsequent waves of follower MLF axons extended from the nucMLF cells and grew along the leading axon (Fig. 5B) (supplemental video 1, available at www.jneurosci.org as supplemental material). Growth cones of follower axons rarely sampled the surrounding environment and instead remained in contact with the leading axon. The growth cones of leading and follower MLF axons were indistinguishable in morphology. In embryos injected with Sema3DMO or L1.1MO, the leading MLF axon extended from the caudalmost nucMLF cell and its growth cone behaved similarly to those in the uninjected controls. However, knockdown of Sema3D or L1.1 disrupted interactions between the growth cones of follower MLF axons and the leading axon (Fig. 5C,D) (supplemental video 2, available at www.jneurosci.org as supplemental material). Follower axons did not maintain stable contacts with the leading axon in the absence of Sema3D or L1.1. Instead, the growth cones of defasciculated follower axons transiently contacted the leading axon and then retracted before growing in parallel with the leader rather than along it (Fig. 5C). A more extreme example is shown after L1.1 knockdown (Fig. 5D), in which the growth cone of a defasciculated follower axon repeatedly contacted and then retracted from the leading axon but failed to ever extend. Furthermore, between 11\% (Sema3DMO) and 14\% (L1.1MO) of defasciculated axons made dramatic directional pathfinding errors within our imaging region. These axons extended $>100 \mu \mathrm{m}$ from the leading axon and grew either medially or laterally, with ap- 


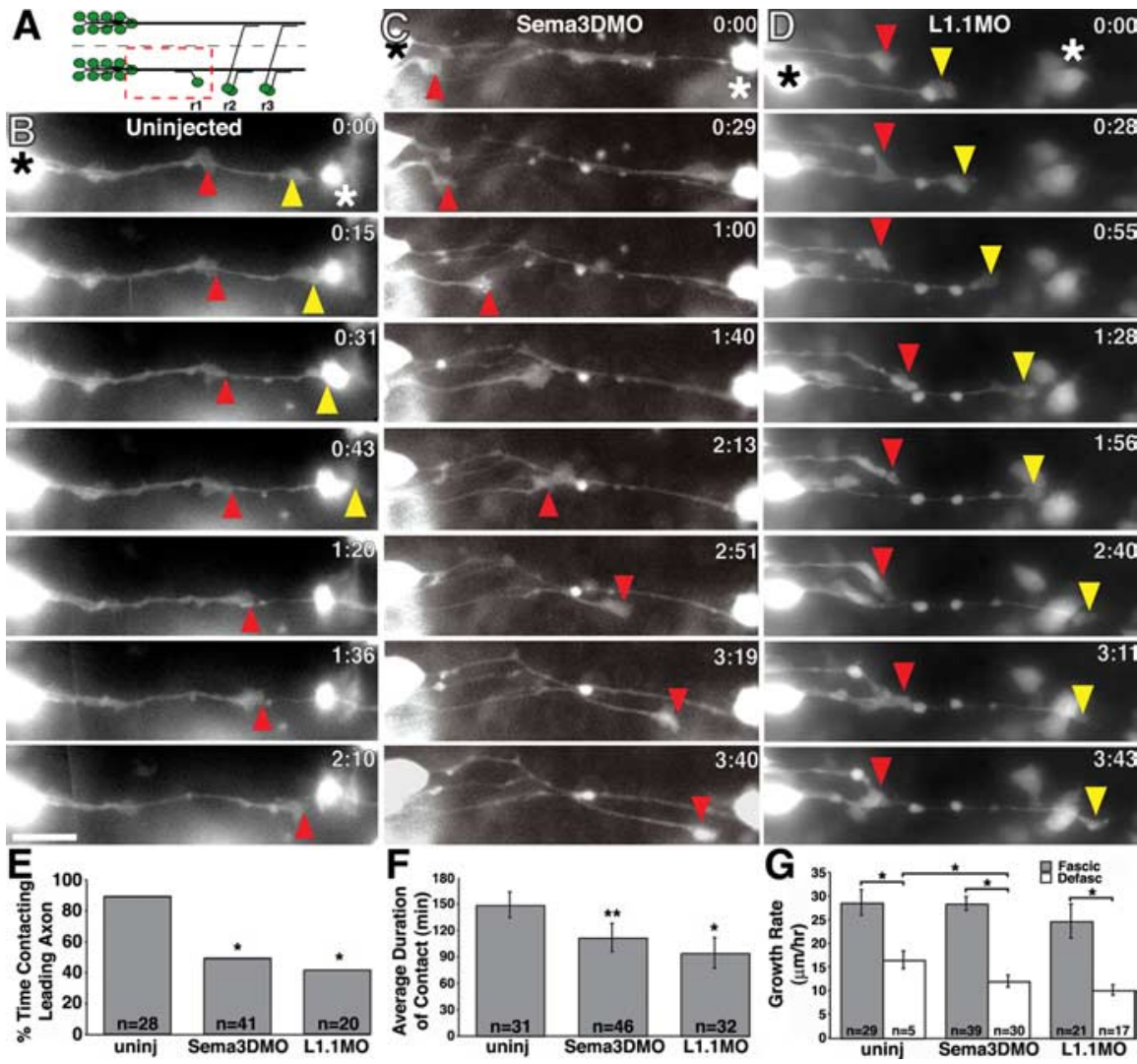

Figure 5. Sema3D and L1.1 regulate dynamic axon-axon interactions among MLF axons. $\boldsymbol{A}$, Schematic representation of imaging area. $\boldsymbol{B}-\boldsymbol{D}$, Images from time-lapse sequence of MLF axon growth in uninjected (B), Sema3DM0-injected ( $\boldsymbol{C}$, or L1.1M0injected (D) Tg(huc:gfp) embryos. Ventral views, anterior to the left. The black asterisk denotes caudalmost nucMLF cell that extends leading MLF axon (yellow arrowheads). Red arrowheads indicate growth cones of follower MLF axons. The white asterisk denotes r1. Time stamp shows hours:minutes. $\boldsymbol{E}$, Quantification of the mean percentage of time in which follower axons spend contacting the leading axon. ${ }^{*} p<0.001$, two-sample binomial comparison. $\boldsymbol{F}$, Quantification of the average duration of each contact between follower growth cone and leading axon. $G$, Quantification of average growth rates of fasciculated and defasciculated follower axons. ${ }^{*} p<0.01,{ }^{* *} p<0.05$, two-tailed $t$ test. Error bars represent SEM. Scale bar, $25 \mu \mathrm{m}$.

proximately the same frequency (data not shown). To quantify the preference of follower growth cones to adhere to the leading axon, we measured the overall percentage of time that follower growth cones contacted the leading axon and the average duration of individual contacts (Fig. $5 E, F$ ). Follower axons spent less overall time in contact with the leading axon (Fig. $5 E$ ), and, when follower growth cones were in contact with the leading axon, their average duration of contact was significantly shorter (Fig. $5 F$ ) after Sema3D or L1.1 knockdown. Collectively, these observations suggest that follower growth cones with normal L1.1 levels maintain their interaction with the leader, but those expressing reduced L1.1 break contact with the leader more readily. The data also suggest that, in the absence of Sema3D or L1.1, follower growth cones no longer find the leading axon as favorable a substrate on which to extend. Moreover, the indistinguishable aberrations in growth cone behavior after Sema3D or L1.1 knockdown supports the hypothesis that the function of Sema3D is to regulate L1.1 levels.

We calculated the growth rates of leading MLF axons and fasciculated or defasciculated follower axons in uninjected, Sema3DMO-, and L1.1MO-injected embryos (Fig. 5G). In each group, leading MLF axons grew at approximately similar rates, suggesting that the growth cone of the leading MLF axon interprets the tissue similarly whether or not the growth cone expresses L1.1 or is in the presence of Sema3D (data not shown).
Also, fasciculated follower axons grew at approximately the same rate in all groups. However, in each group, defasciculated axons grew significantly slower than fasciculated axons. Interestingly, defasciculated follower axons grew more slowly after Sema3D knockdown than the rare but naturally occurring defasciculated axons in the uninjected group. This finding is again inconsistent with the idea that Sema3D promotes MLF fasciculation via repulsion from the surrounding tissue, because we would have expected defasciculated MLF axons to increase their growth rate in the absence of the potentially repulsive Sema3D. Instead, these results suggest that the loss of Sema3D or L1.1 function cause similar specific defects in axon-axon interactions.

\section{Sema3D and L1.1 promote axon-axon} interactions between hindbrain neurons and MLF axons

In the course of our live imaging experiments, we discovered that Sema3D or L1.1 knockdown also affects interactions between hindbrain axons and the MLF. In the hindbrain, neurons positioned lateral to the MLF extend axons that fasciculate along the ipsilateral or contralateral MLF en route to their targets (Fig. 5A). We analyzed the dynamic behaviors and pathfinding decisions of a previously uncharacterized neuron positioned within a three-neuron cluster located in $\mathrm{r} 1(\mathrm{r} 1 \mathrm{~N})$. In uninjected $T g(h u c: g f p)$ embryos, the r1N axon first extended medially toward the MLF. On contacting the MLF, the r1N growth cone turned rostrally and fasciculated along the MLF (Fig. $6 A$ ). Knockdown of either Sema3D or L1.1 reduced fasciculation between the r1N axon and the MLF and caused directional pathfinding errors by $r 1 N$. An example in Figure $6 \mathrm{~B}$ shows that, after Sema3D knockdown, the r1N growth cone contacted the MLF, retracted, and then extended caudally instead of rostrally. Overall, knockdown of Sema3D or L1.1 increased the incidence of defasciculation of the $1 \mathrm{~N}$ axon with the MLF and the prevalence of directional guidance errors compared with r1N axons in uninjected $T g(h u c: g f p)$ embryos (Fig. 6C). Again, the similarity of the defects in r1N axon pathfinding after knockdown of either Sema3D or L1.1 is consistent with a model for Sema3D and L1.1 functioning cooperatively to promote axon-axon interactions.

\section{Sema3D can increase L1.1 levels and hyperfasciculate anterior} commissure axons

To test the ability of Sema3D to regulate L1.1 and fasciculation independent of potential repulsive effects, we analyzed fasciculation in AC axons. We have shown previously that Sema3D is attractive to AC axons (Wolman et al., 2004). Unlike the MLF, $\mathrm{AC}$ axons are loosely fasciculated, allowing us to test whether overexpression of Sema3D or L1.1 causes hyperfasciculation. Both heat-induced Sema3D overexpression in $T g\left(h s p: s e m a 3 d^{m y c}\right)$ embryos (hs-Sema3D) and L1.1 overexpression by mRNA injection significantly reduced the width of the AC at the ventral mid- 


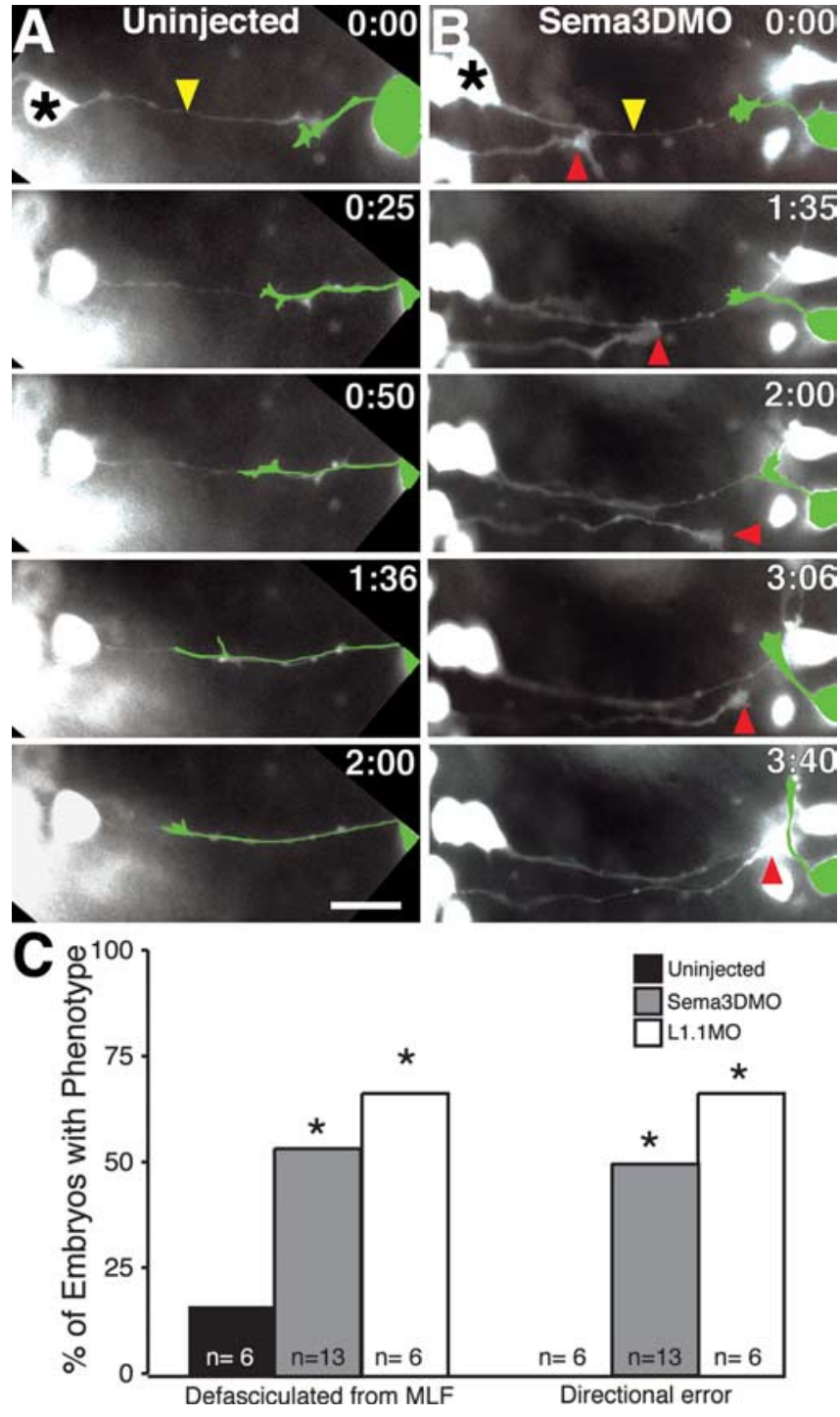

Figure 6. Sema3D influences interactions between MLF axons and hindbrain neurons. $\boldsymbol{A}, \boldsymbol{B}$, Images from time-lapse sequence of interaction between MLF axons and the r1N axon in uninjected $(\boldsymbol{A})$ and Sema3DM0-injected (B) $\operatorname{Tg}$ (huc:gfp) embryos. Ventral views, anterior to the left. The black asterisk indicates caudalmost nucMLF cell that extends leading MLF axon (yellow arrowhead). Red arrowheads denotes growth cone of a defasciculated MLF axon. r1N neuron is shaded in green. Time stamp shows hours:minutes. C, Quantification of the percentage of embryos in which the r1N axon fasciculates with the MLF and grows rostrally. ${ }^{*} p<0.01$, two-sample binomial comparison. Scale bar, $25 \mu \mathrm{m}$.

line versus controls (Fig. $7 A-C$ ), suggesting that overexpression of Sema3D or L1.1 can enhance AC fasciculation. However, L1.1 knockdown prevented Sema3D overexpression from causing AC hyperfasciculation, suggesting that Sema3D acts via L1.1 to cause hyperfasciculation (Fig. 7C). To address whether Sema3D overexpression caused hyperfasciculation of AC axons by increasing L1.1 protein levels on AC axons, we labeled heat-shocked and unheated $\operatorname{Tg}\left(\right.$ hsp:sema3 $\left.d^{\text {myc }}\right)$ embryos with anti-L1.1 and antiacetylated $\alpha$-tubulin antibodies, measured the average fluorescent intensity of each antibody in AC axons from unsaturated confocal projections, and calculated the intensity ratio of L1.1/ tubulin. As shown for MLF axons, Sema3D overexpression significantly increased L1.1 levels in AC axons by $26 \%$ over control levels $(n=31 ; p<0.001)$ (Fig. $7 A, B)$. These results suggest that Sema3D can regulate $\mathrm{L} 1.1$ in multiple axon tracts and thereby influence their fasciculation.

\section{Discussion}

Axon fasciculation can be regulated by interaxonal adhesion and repulsion from surrounding tissues. This study reveals crosstalk between molecules thought to typically function through one or the other of these mechanisms. Classically, repulsive axon guidance molecules, like semaphorins, have been proposed to regulate axon fasciculation by creating a repulsive surround that drives axons together. Elimination of these cues, or their neuronally expressed receptors, causes axons to defasciculate and innervate the normally inhibitory tissue. Although we have shown that Sema3D can be repulsive to growing axons in vivo (Liu et al., 2004; Wolman et al., 2004; Liu and Halloran, 2005), the domain of Sema3D expression (Halloran et al., 1999; Wolman et al., 2004), coupled with the pattern of MLF defasciculation after Sema3D knockdown and the inability of ectopic Sema3D to repel MLF axons in the hindbrain, led us to investigate alternative mechanisms by which Sema3D might regulate MLF axon fasciculation. Several studies have shown that repulsive semaphorins can regulate integrin activity and thereby influence adhesive forces between cells and the extracellular matrix (Serini et al. 2003; Kruger et al., 2005; Pasterkamp, 2005; Halloran and Wolman, 2006). Here, we propose that semaphorins can also modulate adhesion molecule expression on axons to regulate axonaxon interactions. In support of this idea, we show that Sema3D regulates axonal L1.1 protein levels to promote fasciculation in the developing zebrafish brain. Using in vivo time-lapse imaging, we showed a reduced affinity of MLF axons for each other and for specific hindbrain axons after Sema3D or L1.1 knockdown, defects that are consistent with a loss of adhesion. These defects disrupted normal axon-axon interactions that are required for fasciculation and proper pathfinding decisions.

Interaxonal adhesion and repulsion are critical for regulating axon fasciculation and defasciculation. Our live imaging experiments clearly show that the growth cones of follower MLF axons interpret the leading MLF axon differently after loss of Sema3D or L1.1. Rather than extending along the leading axon, growth cones of defasciculated axons transiently contact the leading axon but fail to maintain this contact and do not extend via the prelaid tract. Given the role for Sema3D in regulating L1.1 described here and the well documented role of L1 in mediating cell adhesion, it is possible that the failure of follower growth cones to maintain their contact with the leading tract is attributable to insufficient adhesion. However, it is also possible that the axons defasciculate because knockdown of Sema3D or L1.1 unmasks an axonal molecule that mediates mutual repulsion among MLF axons. In support of this idea, we routinely observed growth cones of defasciculated MLF axons or $\mathrm{r} 1 \mathrm{~N}$ axons retracting after transient contact with the leading axon in embryos lacking Sema3D or L1.1. This possibility may indicate that Sema3D and L1.1 normally function to inhibit premature defasciculation. Independent of whether the regulation by Sema3D of L1.1 functions to increase adhesion or to mask interaxonal repulsion of fasciculating axons or both, it is clear that upsetting axon-axon interactions can disrupt fasciculation and lead to pathfinding errors.

One open question is the mechanism by which Sema3D binds and signals to regulate L1.1 protein levels. Surprisingly, our results suggest that this regulation might be Neuropilin independent because Nrp1A knockdown did not reduce MLF L1.1 protein levels, nor did it preclude Sema3D overexpression from increasing MLF L1.1 protein levels. Moreover, Sema3D overexpression rescued defasciculation caused by Nrp1A knockdown. However, it remains possible that Nrp1A knockdown was not 


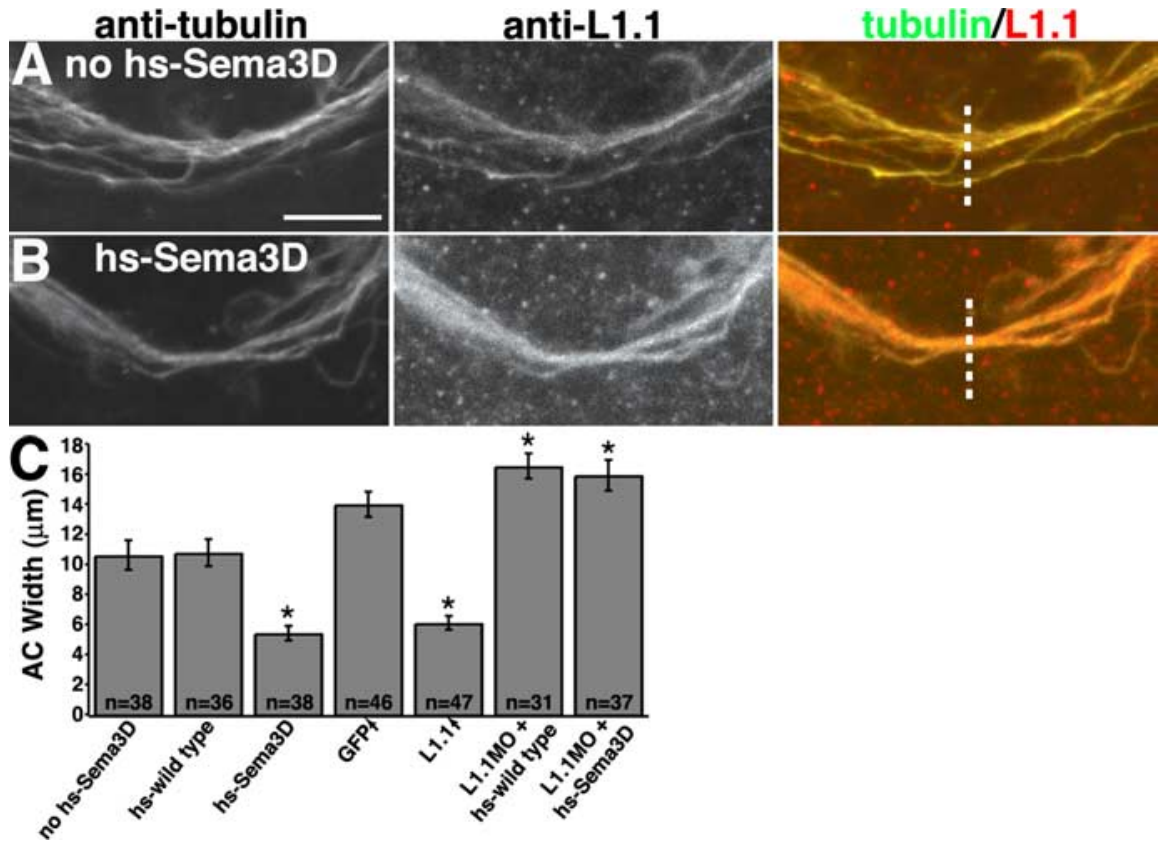

Figure 7. Regulation of $L 1.1$ by Sema3D influences $A C$ axon fasciculation. $\boldsymbol{A}, \boldsymbol{B}$, Unsaturated confocal projections of $27 \mathrm{hpf}$ embryos, labeled with anti-acetylated $\alpha$-tubulin (green) and L1.1 (red) antibodies. Dorsal is up. The dashed line denotes midline. $\boldsymbol{A}$, Unheated $T g$ (hsp:sema3 ${ }^{\text {myc }}$ ) embryo (no hs-Sema3D) shows typical loosely fasciculated AC axons. $\boldsymbol{B}$, Heat-shocked Tg(hsp:sema3 ${ }^{\text {myc }}$ ) embryo (hs-Sema3D) possesses hyperfasciculated AC axons that express higher levels of L1.1.C, Mean width of AC at the ventral midline. ${ }^{*} p<0.001$, two-tailed $t$ test versus no hs-Sema3D or hs-wild type. Error bars represent SEM. Scale bar, $25 \mu \mathrm{m}$.

complete and that residual, low levels of Nrp1A were sufficient to bind Sema3D and mediate its regulation of L1.1 levels. It is unlikely that Sema3D could signal via an alternative Nrp to regulate MLF L1.1 protein levels because Nrp1A appears to be the only Nrp gene expressed by these neurons (Wolman et al., 2004). Alternatively, Sema3D might regulate L1.1 levels by signaling directly through Plexin. However, knockdown of the known Plexins expressed by the nucMLF did not disrupt MLF fasciculation. Again, it is possible that residual PlexinA3 and/or PlexinA4 remained after morpholino injection and were sufficient to mediate Sema3D regulation of MLF fasciculation and L1.1, or another unidentified Plexin might serve this role either independently or in cooperation with Nrp1A. Finally, we should not exclude the possibility that an unidentified receptor molecule mediates Sema3D regulation of L1.1. Future studies will likely elucidate the signaling pathway by which Sema3D regulates L1.1.

Despite the possibility that Nrp1A does not mediate the regulation by Sema3D of L1.1, the genetic interactions between Sema3D-Nrp1A and L1.1-Nrp1A suggest that Nrp1A cooperates with Sema3D and L1.1 to promote MLF fasciculation. Both L1 and neuropilin have been shown to mediate cell-cell adhesion by binding homophilically and heterophilically to a host of partners, including each other (Fujisawa, 2002; Haspel and Grumet, 2003). Interestingly, it has been proposed that the loss of L1-Nrp1 heterophilic interactions is more critical than the loss of L1 homophilic interactions to the axon guidance defects observed in the L1 knock-out mouse (Itoh et al., 2004). Therefore, one possible function for Nrp1A is to bind L1.1 in trans to mediate axonaxon interactions in the presence of Sema3D. Our data showing that Nrp1A overexpression does not rescue defasciculation caused by L1.1 knockdown suggests that Nrp1A homophilic interactions cannot sufficiently compensate for potential L1.1Nrp1A heterophilic interactions. This idea is further supported by our observation that simultaneous partial or full knockdown of Nrp1A and L1.1 elicits the most robust degree of defasciculation (data not shown). However, given the defasciculation rescue by either Sema3D or L1.1 overexpression after Nrp1A knockdown, Nrp1A is not the only critical binding partner of L1.1 important for mediating fasciculation.

Although originally described as repulsive guidance cues, class 3 Semaphorins have also been shown to function as attractive signals and now modulators of adhesion. Interestingly, Sema3D appears to play multiple guidance roles in the pathfinding of a single population of neurons, the zebrafish nucMLF. Previously, we showed that Sema3D initially repels nucMLF axons caudally from their cell bodies in the midbrain via Nrp1A (Wolman et al., 2004), and here we show that MLF axons are not repelled by Sema3D once they extend into the hindbrain, suggesting that MLF axons respond differently to Sema3D at specific stages of their outgrowth. How this differential responsiveness is regulated remains to be determined. One possibility is that Sema3D has distinct effects on the cell body and initial emerging axon versus the growth cone and axon shaft in the hindbrain, perhaps because different signaling components are present in these different neuronal compartments. Another possibility is that repulsion by Sema3D might be mitigated by other factors in the hindbrain, such as attractive molecules that promote growth through the hindbrain or suppressors of repulsion, such as stromal cell-derived factor-1 (Chalasani et al., 2003). Responsiveness may also be regulated intracellularly by cyclic nucleotide levels (Song and Poo, 1999; Polleux et al., 2000). Alternatively, cell surface Nrp1A, which is required for Sema3D repulsion, may be unable to bind extracellular Sema3D in the hindbrain because it may be bound homophilically or to other adhesion molecules, like L1, on neighboring axons to promote fasciculation. Although how the change in responsiveness is regulated remains unknown, these studies reveal the complexity of the influence of Sema3D on MLF pathfinding.

In conclusion, we describe a novel mechanism by which a class 3 semaphorin regulates axon fasciculation. Our results show that semaphorins, which have been proposed previously to promote fasciculation by creating a repulsive surround, may regulate fasciculation by modulating axon-axon interactions in addition to, or possibly instead of, repulsive action.

\section{References}

Bastiani MJ, Raper JA, Goodman CS (1984) Pathfinding by neuronal growth cones in grasshopper embryos. III. Selective affinity of the G growth cone for the $\mathrm{P}$ cells within the $\mathrm{A} / \mathrm{P}$ fascicle. $\mathrm{J}$ Neurosci 4:2311-2328

Becker CG, Schweitzer J, Feldner J, Schachner M, Becker T (2004) Tenascin- $\mathrm{R}$ as a repellent guidance molecule for newly growing and regenerating optic axons in adult zebrafish. Mol Cell Neurosci 26:376-389.

Beer S, Oleszewski M, Gutwein P, Geiger C, Altevogt P (1999) Metalloproteinase-mediated release of the ectodomain of $\mathrm{L} 1$ adhesion molecule. J Cell Sci 112:2667-2675.

Brummendorf T, Kenwrick S, Rathjen FG (1998) Neural cell recognition 
molecule L1: from cell biology to human hereditary brain malformations. Curr Opin Neurobiol 8:87-97.

Castellani V, Chedotal A, Schachner M, Faivre-Sarrailh C, Rougon G (2000) Analysis of the L1-deficient mouse phenotype reveals cross-talk between Sema3A and L1 signaling pathways in axonal guidance. Neuron 27:237-249.

Castellani V, De Angelis E, Kenwrick S, Rougon G (2002) Cis and trans interactions of L1 with neuropilin-1 control axonal responses to semaphorin 3A. EMBO J 21:6348-6357.

Castellani V, Falk J, Rougon G (2004) Semaphorin3A-induced receptor endocytosis during axon guidance responses is mediated by L1 CAM. Mol Cell Neurosci 26:89-100.

Chalasani SH, Sabelko KA, Sunshine MJ, Littman DR, Raper JA (2003) A chemokine, SDF-1, reduces the effectiveness of multiple axonal repellents and is required for normal axon pathfinding. J Neurosci 23:1360-1371.

Chen ZY, Sun C, Reuhl K, Bergemann A, Henkemeyer M, Zhou R (2004) Abnormal hippocampal axon bundling in EphB receptor mutant mice. J Neurosci 24:2366-2374.

Cloutier JF, Giger RJ, Koentges G, Dulac C, Kolodkin AL, Ginty DD (2002) Neuropilin-2 mediates axonal fasciculation, zonal segregation, but not axonal convergence, of primary accessory olfactory neurons. Neuron 33:877-892.

Cloutier JF, Sahay A, Chang EC, Tessier-Lavigne M, Dulac C, Kolodkin AL, Ginty DD (2004) Differential requirements for semaphorin 3F and Slit-1 in axonal targeting, fasciculation, and segregation of olfactory sensory neuron projections. J Neurosci 24:9087-9096.

Cox KH, DeLeon DV, Angerer LM, Angerer RC (1984) Detection of mrnas in sea urchin embryos by in situ hybridization using asymmetric RNA probes. Dev Biol 101:485-502.

Feldner J, Reimer MM, Schweitzer J, Wendik B, Meyer D, Becker T, Becker CG (2007) PlexinA3 restricts spinal exit points and branching of trunk motor nerves in embryonic zebrafish. J Neurosci 27:4978-4983.

Fujisawa H (2002) From the discovery of neuropilin to the determination of its adhesion sites. Adv Exp Med Biol 515:1-12.

Fujisawa H, Kitsukawa T, Kawakami A, Takagi S, Shimizu M, Hirata T (1997) Roles of a neuronal cell-surface molecule, neuropilin, in nerve fiber fasciculation and guidance. Cell Tissue Res 290:465-470.

Giger RJ, Cloutier JF, Sahay A, Prinjha RK, Levengood DV, Moore SE, Pickering S, Simmons D, Rastan S, Walsh FS, Kolodkin AL, Ginty DD, Geppert M (2000) Neuropilin-2 is required in vivo for selective axon guidance responses to secreted semaphorins. Neuron 25:29-41.

Gu C, Yoshida Y, Livet J, Reimert DV, Mann F, Merte J, Henderson CE, Jessell TM, Kolodkin AL, Ginty DD (2005) Semaphorin 3E and plexin-D1 control vascular pattern independently of neuropilins. Science 307:265-268.

Halloran MC, Wolman MA (2006) Repulsion or adhesion: receptors make the call. Curr Opin Cell Biol 18:533-540.

Halloran MC, Severance SM, Yee CS, Gemza DL, Raper JA, Kuwada JY (1999) Analysis of a zebrafish semaphorin reveals potential functions in vivo. Dev Dyn 214:13-25.

Haspel J, Grumet M (2003) The L1CAM extracellular region: a multidomain protein with modular and cooperative binding modes. Front Biosci 8:s1210-s1225.

Huber AB, Kania A, Tran TS, Gu C, De Marco Garcia N, Lieberam I, Johnson D, Jessell TM, Ginty DD, Kolodkin AL (2005) Distinct roles for secreted semaphorin signaling in spinal motor axon guidance. Neuron 48:949-964.

Hutson LD, Chien CB (2002) Pathfinding and error correction by retinal axons: the role of astray/robo2. Neuron 33:205-217.

Isbister CM, Tsai A, Wong ST, Kolodkin AL, O'Connor TP (1999) Discrete roles for secreted and transmembrane semaphorins in neuronal growth cone guidance in vivo. Mol Cell Neurosci 13:143-166.

Itoh K, Cheng L, Kamei Y, Fushiki S, Kamiguchi H, Gutwein P, Stoeck A, Arnold B, Altevogt P, Lemmon V (2004) Brain development in mice lacking L1-L1 homophilic adhesion. J Cell Biol 165:145-154.

Kaneko M, Nighorn A (2003) Interaxonal Eph-ephrin signaling may mediate sorting of olfactory sensory axons in Manduca sexta. J Neurosci 23:11523-11538.

Karagogeos D (2003) Neural GPI-anchored cell adhesion molecules. Front Biosci 8:s1304-s1320.

Kimmel CB, Ballard WW, Kimmel SR, Ullmann B, Schilling TF (1995) Stages of embryonic development of the zebrafish. Dev Dyn 203:253-310.
Kitsukawa T, Shimizu M, Sanbo M, Hirata T, Taniguchi M, Bekku Y, Yagi T, Fujisawa H (1997) Neuropilin-semaphorin III/D-mediated chemorepulsive signals play a crucial role in peripheral nerve projection in mice. Neuron 19:995-1005.

Klose M, Bentley D (1989) Transient pioneer neurons are essential for formation of an embryonic peripheral nerve. Science 245:982-984.

Kruger RP, Aurandt J, Guan KL (2005) Semaphorins command cells to move. Nat Rev Mol Cell Biol 6:789-800.

Kuan YS, Yu HH, Moens CB, Halpern ME (2007) Neuropilin asymmetry mediates a left-right difference in habenular connectivity. Development 134:857-865.

Kuwada JY (1986) Cell recognition by neuronal growth cones in a simple vertebrate embryo. Science 233:740-746.

Langenberg T, Brand M, Cooper MS (2003) Imaging brain development and organogenesis in zebrafish using immobilized embryonic explants. Dev Dyn 228:464-474.

Lin DM, Goodman CS (1994) Ectopic and increased expression of Fasciclin II alters motoneuron growth cone guidance. Neuron 13:507-523.

Lin DM, Fetter RD, Kopczynski C, Grenningloh G, Goodman CS (1994) Genetic analysis of Fasciclin II in Drosophila: defasciculation, refasciculation, and altered fasciculation. Neuron 13:1055-1069.

Liu Y, Halloran MC (2005) Central and peripheral axon branches from one neuron are guided differentially by Semaphorin3D and transient axonal glycoprotein-1. J Neurosci 25:10556-10563.

Liu Y, Berndt J, Su F, Tawarayama H, Shoji W, Kuwada JY, Halloran MC (2004) Semaphorin3D guides retinal axons along the dorsoventral axis of the tectum. J Neurosci 24:310-318.

Mechtersheimer S, Gutwein P, Agmon-Levin N, Stoeck A, Oleszewski M, Riedle S, Postina R, Fahrenholz F, Fogel M, Lemmon V, Altevogt P (2001) Ectodomain shedding of L1 adhesion molecule promotes cell migration by autocrine binding to integrins. J Cell Biol 155:661-673.

Miyashita T, Yeo SY, Hirate Y, Segawa H, Wada H, Little MH, Yamada T, Takahashi N, Okamoto H (2004) PlexinA4 is necessary as a downstream target of Islet2 to mediate Slit signaling for promotion of sensory axon branching. Development 131:3705-3715.

Nasevicius A, Ekker SC (2000) Effective targeted gene "knockdown" in zebrafish. Nat Genet 26:216-220.

Nayeem N, Silletti S, Yang X, Lemmon VP, Reisfeld RA, Stallcup WB, Montgomery AM (1999) A potential role for the plasmin(ogen) system in the posttranslational cleavage of the neural cell adhesion molecule L1. J Cell Sci 112:4739-4749.

Ohta K, Mizutani A, Kawakami A, Murakami Y, Kasuya Y, Takagi S, Tanaka H, Fujisawa H (1995) Plexin: a novel neuronal cell surface molecule that mediates cell adhesion via a homophilic binding mechanism in the presence of calcium ions. Neuron 14:1189-1199.

Oster SF, Bodeker MO, He F, Sretavan DW (2003) Invariant Sema5A inhibition serves an ensheathing function during optic nerve development. Development 130:775-784.

Park HC, Kim CH, Bae YK, Yeo SY, Kim SH, Hong SK, Shin J, Yoo KW, Hibi M, Hirano T, Miki N, Chitnis AB, Huh TL (2000) Analysis of upstream elements in the $\mathrm{HuC}$ promoter leads to the establishment of transgenic zebrafish with fluorescent neurons. Dev Biol 227:279-293.

Pasterkamp RJ (2005) R-Ras fills another GAP in semaphorin signalling. Trends Cell Biol 15:61-64.

Polleux F, Morrow T, Ghosh A (2000) Semaphorin 3A is a chemoattractant for cortical apical dendrites. Nature 404:567-573.

Raper JA, Bastiani MJ, Goodman CS (1984) Pathfinding by neuronal growth cones in grasshopper embryos. IV. The effects of ablating the A and $\mathrm{P}$ axons upon the behavior of the $\mathrm{G}$ growth cone. J Neurosci 4:2329-2345.

Ringstedt T, Braisted JE, Brose K, Kidd T, Goodman C, Tessier-Lavigne M, M. OLDD (2000) Slit inhibition of retinal axon growth and its role in retinal axon pathfinding and innervation patterns in the diencephalon. J Neurosci 20:4983-4991.

Sadoul K, Sadoul R, Faissner A, Schachner M (1988) Biochemical characterization of different molecular forms of the neural cell adhesion molecule L1. J Neurochem 50:510-521.

Serini G, Valdembri D, Zanivan S, Morterra G, Burkhardt C, Caccavari F, Zammataro L, Primo L, Tamagnone L, Logan M, Tessier-Lavigne M, Taniguchi M, Puschel AW, Bussolino F (2003) Class 3 semaphorins control vascular morphogenesis by inhibiting integrin function. Nature 424:391-397. 
Shimizu M, Murakami Y, Suto F, Fujisawa H (2000) Determination of cell adhesion sites of neuropilin-1. J Cell Biol 148:1283-1293.

Song HJ, Poo MM (1999) Signal transduction underlying growth cone guidance by diffusible factors. Curr Opin Neurobiol 9:355-363.

Tang J, Landmesser L, Rutishauser U (1992) Polysialic acid influences specific pathfinding by avian motoneurons. Neuron 8:1031-1044.

Tang J, Rutishauser U, Landmesser L (1994) Polysialic acid regulates growth cone behavior during sorting of motor axons in the plexus region. Neuron 13:405-414.

Taniguchi M, Yuasa S, Fujisawa H, Naruse I, Saga S, Mishina M, Yagi T (1997) Disruption of semaphorin III/D gene causes severe abnormality in peripheral nerve projection. Neuron 19:519-530.

Tongiorgi E, Bernhardt RR, Schachner M (1995) Zebrafish neurons express two L1-related molecules during early axonogenesis. J Neurosci Res 42:547-561.

Torres-Vazquez J, Gitler AD, Fraser SD, Berk JD, Pham VN, Fishman MC,
Childs S, Epstein JA, Weinstein BM (2004) Semaphorin-plexin signaling guides patterning of the developing vasculature. Dev Cell 7:117-123.

Tosney KW, Landmesser LT (1985a) Specificity of early motoneuron growth cone outgrowth in the chick embryo. J Neurosci 5:2336-2344.

Tosney KW, Landmesser LT (1985b) Development of the major pathways for neurite outgrowth in the chick hindlimb. Dev Biol 109:193-214.

Wolman MA, Liu Y, Tawarayama H, Shoji W, Halloran MC (2004) Repulsion and attraction of axons by Semaphorin3D are mediated by different neuropilins in vivo. J Neurosci 24:8428-8435.

Yu HH, Araj HH, Ralls SA, Kolodkin AL (1998) The transmembrane Semaphorin Sema I is required in Drosophila for embryonic motor and CNS axon guidance. Neuron 20:207-220.

Yu HH, Huang AS, Kolodkin AL (2000) Semaphorin-la acts in concert with the cell adhesion molecules fasciclin II and connectin to regulate axon fasciculation in Drosophila. Genetics 156:723-731. 